\title{
Observation of $B^{+} \rightarrow \Lambda_{c}^{+} \Lambda_{c}^{-} K^{+}$and $B^{0} \rightarrow \Lambda_{c}^{+} \Lambda_{c}^{-} K^{0}$ decays
}

N. Gabyshev, ${ }^{1}$ K. Abe ${ }^{6}$ K. Abe,${ }^{37}$ I. Adachi, ${ }^{6}$ H. Aihara, ${ }^{39}$ Y. Asano, ${ }^{42}$ V. Aulchenko, ${ }^{1}$ T. Aushev, ${ }^{10}$ S. Bahinipati, ${ }^{3}$ A. M. Bakich, ${ }^{34}$ V. Balagura, ${ }^{10}$ E. Barberio, ${ }^{17}$ W. Bartel, ${ }^{4}$ A. Bay ${ }^{14}$ I. Bedny,${ }^{1}$ U. Bitenc, ${ }^{11}$ I. Bizjak, ${ }^{11}$ A. Bondar, ${ }^{1}$ A. Bozek, ${ }^{23}$ M. Bračko, ${ }^{6,16,11}$ T. E. Browder, ${ }^{5}$ A. Chen, ${ }^{20}$ W. T. Chen, ${ }^{20}$ B. G. Cheon, ${ }^{2}$ R. Chistov, ${ }^{10}$ Y. Choi,${ }^{33}$ A. Chuvikov, ${ }^{29}$ S. Cole, ${ }^{34}$ J. Dalseno, ${ }^{17}$ M. Danilov, ${ }^{10}$ M. Dash, ${ }^{43}$ A. Drutskoy ${ }^{3}$ S. Eidelman, ${ }^{1}$ A. Garmash, ${ }^{29}$ T. Gershon, ${ }^{6}$ G. Gokhroo, ${ }^{35}$ B. Golob $,{ }^{15}, 11$ J. Haba, ${ }^{6}$ K. Hayasaka, ${ }^{18}$ H. Hayashii, ${ }^{19}$ M. Hazumi, ${ }^{6}$ T. Hokuue, ${ }^{18}$ Y. Hoshi,${ }^{37}$ S. Hou, ${ }^{20}$ W.-S. Hou, ${ }^{22}$ Y. B. Hsiung, ${ }^{22}$ K. Ikado, ${ }^{18}$ A. Imoto,${ }^{19}$ K. Inami, ${ }^{18}$ R. Itoh,${ }^{6}$ M. Iwasaki, ${ }^{39}$ Y. Iwasaki, ${ }^{6}$ J. H. Kang, ${ }^{44}$ T. Kawasaki, ${ }^{24}$ H. R. Khan, ${ }^{40}$ H. Kichimi, ${ }^{6}$ S. M. Kim, ${ }^{33}$ S. Korpar, ${ }^{16,11}$ P. Krokovny, ${ }^{1}$ R. Kulasiri, ${ }^{3}$ C. C. Kuo, ${ }^{20}$ A. Kuzmin, ${ }^{1}$ Y.-J. Kwon, ${ }^{44}$ G. Leder, ${ }^{9}$ T. Lesiak, ${ }^{23}$ S.-W. Lin, ${ }^{22}$ D. Liventsev, ${ }^{10}$ G. Majumder,${ }^{35}$ T. Matsumoto, ${ }^{41}$ W. Mitaroff, ${ }^{9}$ K. Miyabayashi, ${ }^{19}$ H. Miyata, ${ }^{24}$ Y. Miyazaki, ${ }^{18}$ R. Mizuk, ${ }^{10}$ E. Nakano, ${ }^{26}$ M. Nakao, ${ }^{6}$ Z. Natkaniec, ${ }^{23}$ S. Nishida, ${ }^{6}$ S. Ogawa, ${ }^{36}$ T. Ohshima, ${ }^{18}$ T. Okabe, ${ }^{18}$ S. Okuno, ${ }^{12}$ S. L. Olsen, ${ }^{5}$ H. Ozaki, ${ }^{6}$ H. Palka, ${ }^{23}$ C. W. Park, ${ }^{33}$ K. S. Park, ${ }^{33}$ R. Pestotnik, ${ }^{11}$ L. E. Piilonen, ${ }^{43}$ Y. Sakai, ${ }^{6}$ N. Sato,${ }^{18}$ N. Satoyama, ${ }^{32}$ T. Schietinger,${ }^{14}$ O. Schneider, ${ }^{14}$ C. Schwanda, ${ }^{9}$ R. Seidl, ${ }^{30}$ K. Senyo, ${ }^{18}$ M. E. Sevior, ${ }^{17}$ M. Shapkin, ${ }^{8}$ H. Shibuya, ${ }^{36}$ A. Somov, ${ }^{3}$ N. Soni, ${ }^{27}$ R. Stamen, ${ }^{6}$ S. Stanič ${ }^{25}$ M. Starič ${ }^{11}$ T. Sumiyoshi, ${ }^{41}$ K. Tamai,${ }^{6}$ N. Tamura, ${ }^{24}$ M. Tanaka, ${ }^{6}$ G. N. Taylor,${ }^{17}$ Y. Teramoto,${ }^{26}$ X. C. Tian,${ }^{28}$ T. Tsukamoto, ${ }^{6}$ S. Uehara, ${ }^{6}$ T. Uglov, ${ }^{10} \mathrm{~K}$. Ueno, ${ }^{22}$ S. Uno,${ }^{6}$ P. Urquijo, ${ }^{17}$

G. Varner, ${ }^{5}$ K. E. Varvell, ${ }^{34}$ S. Villa, ${ }^{14}$ C. C. Wang, ${ }^{22}$ C. H. Wang, ${ }^{21}$ Y. Watanabe,${ }^{40}$ E. Won, ${ }^{13}$ Q. L. Xie, ${ }^{7}$ A. Yamaguchi, ${ }^{38}$ M. Yamauchi, ${ }^{6}$ J. Ying, ${ }^{28}$ and Z. P. Zhang ${ }^{31}$

(The Belle Collaboration)

${ }^{1}$ Budker Institute of Nuclear Physics, Novosibirsk

${ }^{2}$ Chonnam National University, Kwangju

${ }^{3}$ University of Cincinnati, Cincinnati, Ohio 45221

${ }^{4}$ Deutsches Elektronen-Synchrotron, Hamburg

${ }^{5}$ University of Hawaii, Honolulu, Hawaii 96822

${ }^{6}$ High Energy Accelerator Research Organization (KEK), Tsukuba 
${ }^{7}$ Institute of High Energy Physics, Chinese Academy of Sciences, Beijing

${ }^{8}$ Institute of High Energy Physics, Protvino

${ }^{9}$ Institute of High Energy Physics, Vienna

${ }^{10}$ Institute for Theoretical and Experimental Physics, Moscow

${ }^{11}$ J. Stefan Institute, Ljubljana

${ }^{12}$ Kanagawa University, Yokohama

${ }^{13}$ Korea University, Seoul

${ }^{14}$ Swiss Federal Institute of Technology of Lausanne, EPFL, Lausanne

${ }^{15}$ University of Ljubljana, Ljubljana

${ }^{16}$ University of Maribor, Maribor

${ }^{17}$ University of Melbourne, Victoria

${ }^{18}$ Nagoya University, Nagoya

${ }^{19}$ Nara Women's University, Nara

${ }^{20}$ National Central University, Chung-li

${ }^{21}$ National United University, Miao Li

${ }^{22}$ Department of Physics, National Taiwan University, Taipei

${ }^{23}$ H. Niewodniczanski Institute of Nuclear Physics, Krakow

${ }^{24}$ Niigata University, Niigata

${ }^{25}$ Nova Gorica Polytechnic, Nova Gorica

${ }^{26}$ Osaka City University, Osaka

${ }^{27}$ Panjab University, Chandigarh

${ }^{28}$ Peking University, Beijing

${ }^{29}$ Princeton University, Princeton, New Jersey 08544

${ }^{30}$ RIKEN BNL Research Center, Upton, New York 11973

${ }^{31}$ University of Science and Technology of China, Hefei

32 Shinshu University, Nagano

${ }^{33}$ Sungkyunkwan University, Suwon

${ }^{34}$ University of Sydney, Sydney NSW

${ }^{35}$ Tata Institute of Fundamental Research, Bombay

${ }^{36}$ Toho University, Funabashi

${ }^{37}$ Tohoku Gakuin University, Tagajo

${ }^{38}$ Tohoku University, Sendai 
${ }^{39}$ Department of Physics, University of Tokyo, Tokyo

${ }^{40}$ Tokyo Institute of Technology, Tokyo

${ }^{41}$ Tokyo Metropolitan University, Tokyo

${ }^{42}$ University of Tsukuba, Tsukuba

${ }^{43}$ Virginia Polytechnic Institute and State University, Blacksburg, Virginia 24061

44 Yonsei University, Seoul

(Dated: Version 12.2.3)

\begin{abstract}
We report first measurements of the doubly charmed baryonic $B$ decays $B \rightarrow \Lambda_{c}^{+} \Lambda_{c}^{-} K$. The $B^{+} \rightarrow \Lambda_{c}^{+} \Lambda_{c}^{-} K^{+}$decay is observed with a branching fraction of $\left(6.5_{-0.9}^{+1.0} \pm 1.1 \pm 3.4\right) \times 10^{-4}$ and a statistical significance of $15.4 \sigma$. The $B^{0} \rightarrow \Lambda_{c}^{+} \Lambda_{c}^{-} K^{0}$ decay is observed with a branching fraction of $\left(7.9_{-2.3}^{+2.9} \pm 1.2 \pm 4.1\right) \times 10^{-4}$ and a statistical significance of $6.6 \sigma$. The branching fraction errors are statistical, systematic, and the error resulting from the uncertainty of the $\Lambda_{c}^{+} \rightarrow p K^{-} \pi^{+}$decay branching fraction. The analysis is based on $357 \mathrm{fb}^{-1}$ of data accumulated at the $\Upsilon(4 S)$ resonance with the Belle detector at the KEKB asymmetric-energy $e^{+} e^{-}$collider.
\end{abstract}

PACS numbers: 14.20.Lq, 14.40.Nd 
Recently a number of studies of single charmed baryon production in $B$ decays have been reported 1, 2, 3, 4]. The measured branching fractions of the two-body single charmed baryon decays $\bar{B}^{0} \rightarrow \Lambda_{c}^{+} \bar{p}$ 3] and $B^{-} \rightarrow \Sigma_{c}^{0}(2455) \bar{p}$ [4] are significantly smaller than theoretical expectations [5, 6, 7], 8]. The multi-body single charmed baryon decays $\bar{B} \rightarrow \Lambda_{c}^{+} \bar{p} \pi(\pi)$ were found to have branching fractions about one order of magnitude larger than the corresponding two-body decays, but still below theoretical predictions. While single charm production proceeds via a $b \rightarrow c \bar{u} d$ quark transition, production of two charmed particles occurs via a $b \rightarrow c \bar{c} s$ transition. In contrast to the single charmed baryon production the two-body doubly charmed baryon $B$ decay $\left.B^{+} \rightarrow \bar{\Xi}_{c}^{0} \Lambda_{c}^{+} \mid 9\right]$ recently observed at Belle has a branching fraction comparable to theoretical predictions [5]. It would be interesting to check whether theory can describe multibody double charmed decays. In this paper we report the first observation of the $B^{+} \rightarrow \Lambda_{c}^{+} \Lambda_{c}^{-} K^{+}$and $B^{0} \rightarrow \Lambda_{c}^{+} \Lambda_{c}^{-} K^{0}$ decays, which are three-body decays that proceed via a $b \rightarrow c \bar{c} s$ transition. Inclusion of charge conjugate states is implicit unless otherwise stated. The analysis is based on a data sample of $357 \mathrm{fb}^{-1}$ accumulated at the $\Upsilon(4 S)$ resonance with the Belle detector at the KEKB asymmetric-energy collider corresponding to $386 \times 10^{6} B \bar{B}$ pairs.

The Belle detector is a large-solid-angle magnetic spectrometer that consists of a silicon vertex detector (SVD), a 50-layer central drift chamber (CDC), an array of aerogel threshold Čerenkov counters (ACC), a barrel-like arrangement of time-of-flight scintillation counters (TOF), and an electromagnetic calorimeter (ECL) comprised of $\mathrm{CsI}(\mathrm{Tl})$ crystals located inside a superconducting solenoid coil that provides a $1.5 \mathrm{~T}$ magnetic field. An iron fluxreturn located outside of the coil is instrumented to detect $K_{I}^{0}$ mesons and to identify muons (KLM). The Belle detector is described in detail elsewhere [10]. Two different inner detector configurations were used. For the first sample of $152 \times 10^{6} B \bar{B}$ pairs, a $2.0 \mathrm{~cm}$ radius beampipe and a 3-layer silicon vertex detector were used; for the latter $234 \times 10^{6} \mathrm{~B} \overline{\mathrm{B}}$ pairs, a $1.5 \mathrm{~cm}$ radius beam-pipe, a 4-layer silicon detector and a small-cell inner drift chamber were used [1]. We use a GEANT based Monte Carlo (MC) simulation to model the response of the detector and determine its acceptance [12].

We detect the $\Lambda_{c}^{+}$via the $\Lambda_{c}^{+} \rightarrow p K^{-} \pi^{+}, p \bar{K}^{0}$ and $\Lambda \pi^{+}$decay channels. When a $\Lambda_{c}^{+}$and $\Lambda_{c}^{-}$are combined as $B$ decay daughters, at least one of $\Lambda_{c}^{ \pm}$is required to have been reconstructed via the $p K^{\mp} \pi^{ \pm}$decay process. For each charged track, the particle identification (PID) information from the CDC, ACC and TOF is used to construct likelihood functions 
$L_{p}, L_{K}$ and $L_{\pi}$ for the proton, kaon and pion assignments, respectively. Likelihood ratios $L_{a} /\left(L_{a}+L_{b}\right)$ are required to be greater than 0.6 to identify a particle as type $a$, where $b$ denotes the other two possible hadron assignments from the three possiblities: proton, kaon and pion. For the main mode $B^{+} \rightarrow \Lambda_{c}^{+} \Lambda_{c}^{-} K^{+}, \Lambda_{c}^{+} \rightarrow p K^{-} \pi^{+}, \Lambda_{c}^{-} \rightarrow \bar{p} K^{+} \pi^{-}$the PID efficiency for the primary $K^{+}$is about $95 \%$. Efficiencies for protons, kaons and pions from $\Lambda_{c}^{+}$ decays are about $98 \%$. The misidentification probability for pions (or kaons) to be identified as kaons (or pions) is less than 5\%. The probability for pions or kaons to be identified as protons is less than $2 \%$. Tracks consistent with an electron or muon hypothesis are rejected. $\mathrm{A} \Lambda_{c}^{+}$candidate is selected if the mass of its decay products is within $0.010 \mathrm{GeV} / c^{2}(2.5 \sigma)$ of the nominal $\Lambda_{c}^{+}$mass.

Neutral kaons are reconstructed in the $K_{S}^{0} \rightarrow \pi^{+} \pi^{-}$decay. Candidate $\Lambda$ baryons are reconstructed in the decay $\Lambda \rightarrow p \pi^{-}$. We apply vertex and mass constrained fits for the $K^{0}$ and $\Lambda$ candidates to improve the momentum resolution. The intersection point of the $K^{0}$ and $\Lambda$ candidate daughter tracks must be displaced from the beam interaction point (IP): the flight distance should be more than $0.5 \mathrm{~mm}$. A $K^{0}$ candidate is selected if the mass of its decay products is within $7.5 \mathrm{MeV} / c^{2}(3 \sigma)$ of the $K^{0}$ mass. A $\Lambda$ candidate is selected if the mass of its decay products is within $2.5 \mathrm{MeV} / c^{2}(2.5 \sigma)$ of the $\Lambda$ mass.

The $B$ candidates are identified using the beam-energy constrained mass $M_{\mathrm{bc}}$ and the mass difference $\Delta M_{B}$. The beam-energy constrained mass is defined as $M_{\mathrm{bc}} \equiv$ $\sqrt{E_{\text {beam }}^{2}-\left(\sum \vec{p}_{i}\right)^{2}}$, where $E_{\text {beam }}$ is the beam energy, and $\vec{p}_{i}$ are the three-momenta of the $B$ meson decay products, all defined in the center-of-mass system (CMS) of the $e^{+} e^{-}$collision. The mass difference is defined as $\Delta M_{B} \equiv M(B)-m_{B}$, where $M(B)$ is the reconstructed mass of the $B$ candidate and $m_{B}$ is the world average $B$ meson mass. The parameter $\Delta M_{B}$ is used instead of the energy difference $\Delta E=\left(\sum E_{i}\right)-E_{\text {beam }}$, where $E_{i}$ is the CMS energy of the $B$ decay products, since $\Delta E$ shows a correlation with $M_{\mathrm{bc}}$, while $\Delta M_{B}$ does not [13]. $M(B)=\sqrt{E(B)^{2}-\left(\sum \vec{p}_{i}\right)^{2}}$, where $E(B)=E\left(\Lambda_{c}^{+}\right)+E\left(\Lambda_{c}^{-}\right)+E(K), E\left(\Lambda_{c}^{+}\right)=\sqrt{\vec{p}_{\Lambda_{c}^{+}}^{2}+m_{\Lambda_{c}^{+}}^{2}}$, $\vec{p}_{\Lambda_{c}^{+}}$is the $\Lambda_{c}^{+}$momentum measured via its decay products and $m_{\Lambda_{c}^{+}}$is the value of the $\Lambda_{c}^{+}$ baryon mass [14]. We select events with $M_{\mathrm{bc}}>5.20 \mathrm{GeV} / c^{2}$ and $\left|\Delta M_{B}\right|<0.20 \mathrm{GeV} / c^{2}$. The prompt $K^{+}$or the reconstructed $K_{S}^{0}$ trajectory and the $\Lambda_{c}^{+} / \Lambda_{c}^{-}$trajectories are required to form a common $B$ decay vertex. If there are multiple candidates in an event, the candidate with the best $\chi_{B}^{2}$ for the $B$ vertex fit is selected. The $B$ vertex fit is performed without additional mass constraints for known particles. 

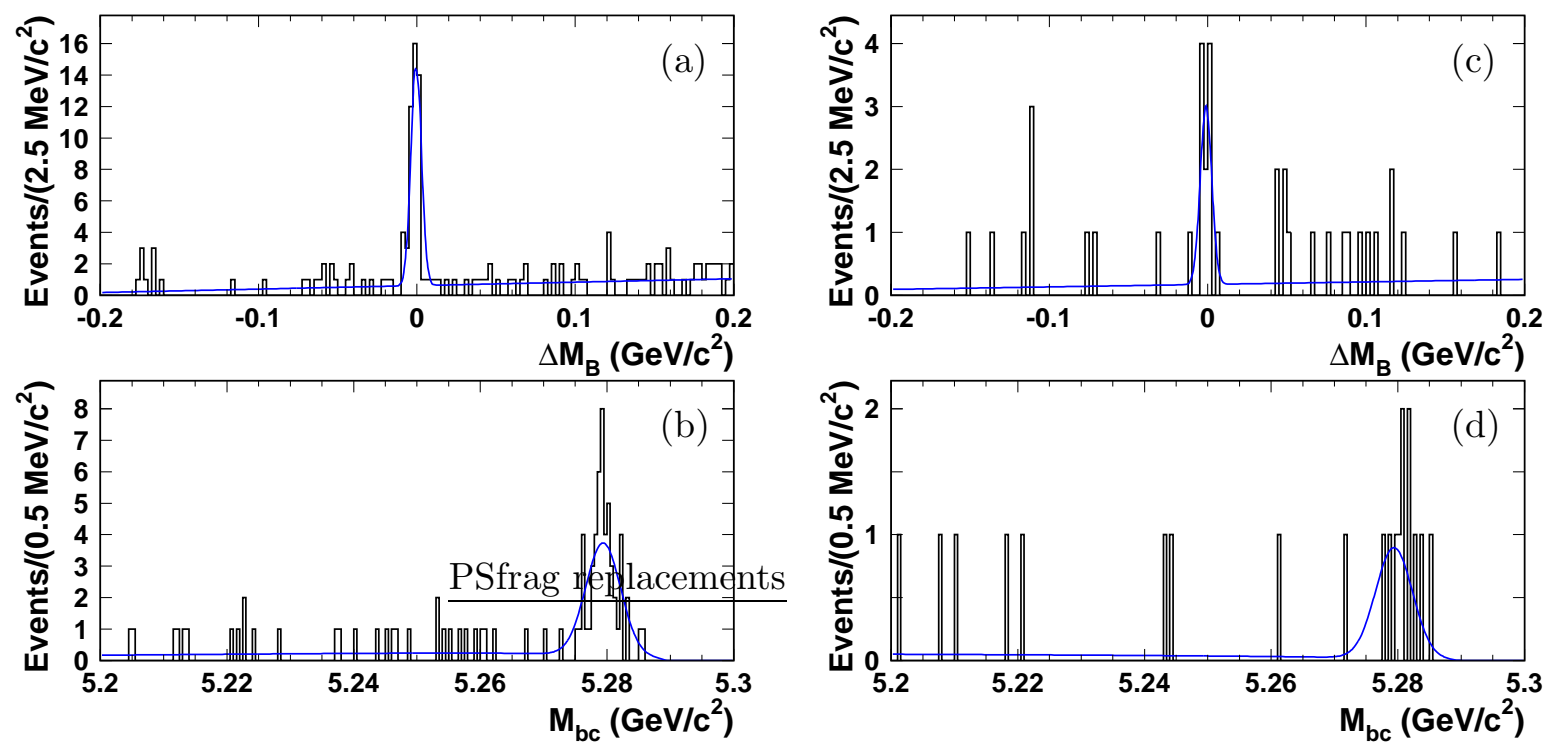

FIG. 1: Candidate (a, b) $B^{+} \rightarrow \Lambda_{c}^{+} \Lambda_{c}^{-} K^{+}$and (c, d) $B^{0} \rightarrow \Lambda_{c}^{+} \Lambda_{c}^{-} K^{0}$ decay events: (a, c) $\Delta M_{B}$ distribution for $M_{\mathrm{bc}}>5.27 \mathrm{GeV} / c^{2}$ and (b, d) $M_{\mathrm{bc}}$ distribution for $\left|\Delta M_{B}\right|<0.015 \mathrm{GeV} / c^{2}$. Curves indicate the fit results.

Figure 1 shows $\Delta M_{B}$ and $M_{\mathrm{bc}}$ projections for selected $B^{+} \rightarrow \Lambda_{c}^{+} \Lambda_{c}^{-} K^{+}$and $B^{0} \rightarrow \Lambda_{c}^{+} \Lambda_{c}^{-} K^{0}$ decay events. The $\Delta M_{B}$ projection is shown for $M_{\mathrm{bc}}>5.27 \mathrm{GeV} / c^{2}$ and the $M_{\mathrm{bc}}$ projection is shown for $\left|\Delta M_{B}\right|<0.015 \mathrm{GeV} / c^{2}$. The widths determined from single Gaussian fits to MC generated events are $2.7 \mathrm{MeV} / c^{2}$ and $3.3 \mathrm{MeV} / c^{2}$ for $M_{\mathrm{bc}}$ and $\Delta M_{B}$, respectively. A two-dimensional binned maximum likelihood fit is performed to determine the signal yield. The $\Delta M_{B}$ distribution is approximated by a Gaussian for the signal plus a first order polynomial for the background, and the $M_{\mathrm{bc}}$ distribution is represented by a single Gaussian for the signal plus an ARGUS function [15] for the background. The signal shape parameters are fixed to the values obtained from a fit to a MC simulation. All yields and background shape parameters are allowed to float.

From the fit we obtain signal yields of $48.5_{-6.8}^{+7.5}$ and $10.5_{-3.1}^{+3.8}$ events with statistical significances of $15.4 \sigma$ and $6.6 \sigma$, for $B^{+} \rightarrow \Lambda_{c}^{+} \Lambda_{c}^{-} K^{+}$and $B^{0} \rightarrow \Lambda_{c}^{+} \Lambda_{c}^{-} K^{0}$, respectively. The significance is calculated as $\sqrt{-2 \ln \left(\mathcal{L}_{0} / \mathcal{L}_{\text {max }}\right)}$, where $\mathcal{L}_{\text {max }}$ and $\mathcal{L}_{0}$ denote the maximum likelihoods with the fitted signal yield and with the yield fixed at zero, respectively.

The branching fraction $\mathcal{B}_{i j}$ for the $i$-th $\Lambda_{c}^{+}$decay and the $j$-th $\Lambda_{c}^{-}$decay mode are calculated as $\mathcal{B}_{i j}=N_{i j} /\left[N_{B \bar{B}} \varepsilon_{i j} \mathcal{B}_{i}\left(\Lambda_{c}^{+}\right) \mathcal{B}_{j}\left(\Lambda_{c}^{-}\right)\right]$, where $N_{i j}$ is the $B$ signal yield. The detection efficiencies, $\varepsilon_{i j}$, are determined from MC simulation. The $\Lambda_{c}^{+}$decay branching 
fractions $\mathcal{B}_{i}\left(\Lambda_{c}^{+}\right)$are converted to the product $\mathcal{B}\left(\Lambda_{c}^{+} \rightarrow p K^{-} \pi^{+}\right) \Gamma_{i} / \Gamma\left(p K^{-} \pi^{+}\right)$to isolate the common uncertainty from the branching fraction of $\Lambda_{c}^{+} \rightarrow p K^{-} \pi^{+}$. The values of $\Gamma_{i} / \Gamma\left(p K^{-} \pi^{+}\right)$are $(0.47 \pm 0.04)$ and $(0.180 \pm 0.032)$ for $p K^{0}$ and $\Lambda \pi^{+}$modes, respectively [16]. The overall detection efficiency $\varepsilon$ for the total signal yield $N$ is calculated as $\sum \varepsilon_{i j}\left[\Gamma_{i} / \Gamma\left(p K^{-} \pi^{+}\right)\right]\left[\Gamma_{j} / \Gamma\left(p K^{-} \pi^{+}\right)\right]$. The overall branching fraction is calculated as $N_{\mathrm{S}} /\left[N_{B \bar{B}} \varepsilon \mathcal{B}\left(\Lambda_{c}^{+} \rightarrow p K^{-} \pi^{+}\right)^{2}\right]$, using the overall signal yield $N_{\mathrm{S}}$ and the decay branching fraction $\mathcal{B}\left(\Lambda_{c}^{+} \rightarrow p K^{-} \pi^{+}\right)=(5.0 \pm 1.3) \%$ [16]. The detection efficiencies are calculated to be $7.79 \%$ for the $B^{+} \rightarrow \Lambda_{c}^{+} \Lambda_{c}^{-} K^{+}$decay and $1.38 \%$ for the $B^{0} \rightarrow \Lambda_{c}^{+} \Lambda_{c}^{-} K^{0}$ decay.

The number of $B \bar{B}$ pairs $N_{B \bar{B}}$ is $(386 \pm 4) \times 10^{6}$. The fractions of charged and neutral $B$ mesons are assumed to be equal. We obtain branching fractions of

$$
\begin{gathered}
\mathcal{B}\left(B^{+} \rightarrow \Lambda_{c}^{+} \Lambda_{c}^{-} K^{+}\right)=\left(6.5_{-0.9}^{+1.0} \pm 1.1 \pm 3.4\right) \times 10^{-4} \text { and } \\
\mathcal{B}\left(B^{0} \rightarrow \Lambda_{c}^{+} \Lambda_{c}^{-} K^{0}\right)=\left(7.9_{-2.3}^{+2.9} \pm 1.2 \pm 4.1\right) \times 10^{-4}
\end{gathered}
$$

where the first and the second errors are statistical and systematic, respectively. The last error is due to the $52 \%$ uncertainty in the absolute branching fraction, $\mathcal{B}\left(\Lambda_{c}^{+} \rightarrow p K^{-} \pi^{+}\right)$.

Systematic uncertainties in the detection efficiencies arise from the track reconstruction efficiency $(8 \%-10 \%$ depending on the process, assuming a correlated systematic error of about $1 \%$ per charged track); the PID efficiency (9\% - 10\% assuming a correlated systematic error of $2 \%$ per proton and $1 \%$ per pion or kaon); 3-body decay model uncertainty (11\% for the $B^{+} \rightarrow \Lambda_{c}^{+} \Lambda_{c}^{-} K^{+}$decay and $5 \%$ for the $B^{0} \rightarrow \Lambda_{c}^{+} \Lambda_{c}^{-} K^{0}$ decay); and MC statistics (1\% $-2 \%)$. The other uncertainties are associated with $\Gamma\left(\Lambda_{c}^{+}\right) / \Gamma\left(p K^{-} \pi^{+}\right)(2 \%-3 \%)$; and the number of $N_{B \bar{B}}$ events (1\%). The total systematic error is $17 \%$ for $B^{+} \rightarrow \Lambda_{c}^{+} \Lambda_{c}^{-} K^{+}$and $15 \%$ for $B^{+} \rightarrow \Lambda_{c}^{+} \Lambda_{c}^{-} K^{0}$.
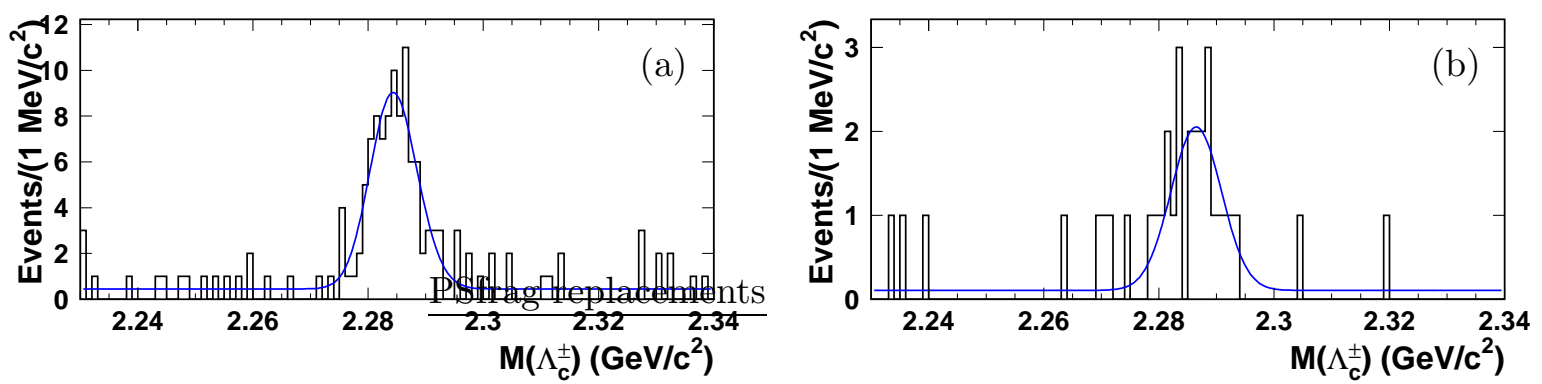

FIG. 2: $\quad M\left(\Lambda_{c}^{ \pm}\right)$mass distributions for (a) $B^{+} \rightarrow \Lambda_{c}^{+} \Lambda_{c}^{-} K^{+}$and (b) $B^{0} \rightarrow \Lambda_{c}^{+} \Lambda_{c}^{-} K^{0}$ decay candidates in the $B$ signal region. Curves indicate the fit results. 
Figure 2 shows the mass distributions $M\left(\Lambda_{c}^{ \pm}\right)$for $B$ candidates in the signal region $\left|\Delta M_{B}\right|<0.015 \mathrm{GeV} / c^{2}$ and $M_{\mathrm{bc}}>5.27 \mathrm{GeV} / c^{2}$. The $M\left(\Lambda_{c}^{ \pm}\right)$mass distributions are shown for $\left|M\left(\Lambda_{c}^{\mp}\right)-m_{\Lambda_{c}^{+}}\right|<0.010 \mathrm{GeV} / c^{2}$. The curves show the results of a fit with the sum of a Gaussian and a linear background. The means and widths of the Gaussians are fixed to values obtained from fits to MC samples. For $B^{+} \rightarrow \Lambda_{c}^{+} \Lambda_{c}^{-} K^{+}$decay, we obtain a $\Lambda_{c}^{+}$yield of $39.5_{-6.5}^{+7.3}$ events and a $\Lambda_{c}^{-}$yield of $48.2_{-7.0}^{+7.7}$ events. For $B^{0} \rightarrow \Lambda_{c}^{+} \Lambda_{c}^{-} K^{0}$, yields of $11.4_{-3.2}^{+3.8}$ and $10.0_{-3.1}^{+3.8}$ events are obtained from the $\Lambda_{c}^{+}$and $\Lambda_{c}^{-}$distributions, respectively. These values are consistent with the $B$ signal yields given above.

We consider possible contributions from other $B$ decays, which could give a $B$ signal in the $\Delta E$ and $\Delta M_{B}$ distributions, but should produce a uniform distribution in the $\Lambda_{c}^{+}$mass region. To assess this type of background, we analyze the $\Lambda_{c}^{+}$sideband $0.015 \mathrm{GeV} / c^{2}<$ $\left|M\left(\Lambda_{c}^{+}\right)-m_{\Lambda_{c}^{+}}\right|<0.055 \mathrm{GeV} / c^{2}$ and $\left|M\left(\Lambda_{c}^{-}\right)-m_{\Lambda_{c}^{-}}\right|<0.010 \mathrm{GeV} / c^{2}$, and $\Lambda_{c}^{-}$sideband $0.015 \mathrm{GeV} / c^{2}<\left|M\left(\Lambda_{c}^{-}\right)-m_{\Lambda_{c}^{-}}\right|<0.055 \mathrm{GeV} / c^{2}$ and $\left|M\left(\Lambda_{c}^{+}\right)-m_{\Lambda_{c}^{+}}\right|<0.010 \mathrm{GeV} / c^{2}$. We conclude that other $B$ decays contribute less than 1.7 events at 90\% C.L. in the $B^{+} \rightarrow \Lambda_{c}^{+} \Lambda_{c}^{-} K^{+}$mode and less than 0.2 events at $90 \%$ C.L. in $B^{0} \rightarrow \Lambda_{c}^{+} \Lambda_{c}^{-} K^{0}$; both contributions are neglected.
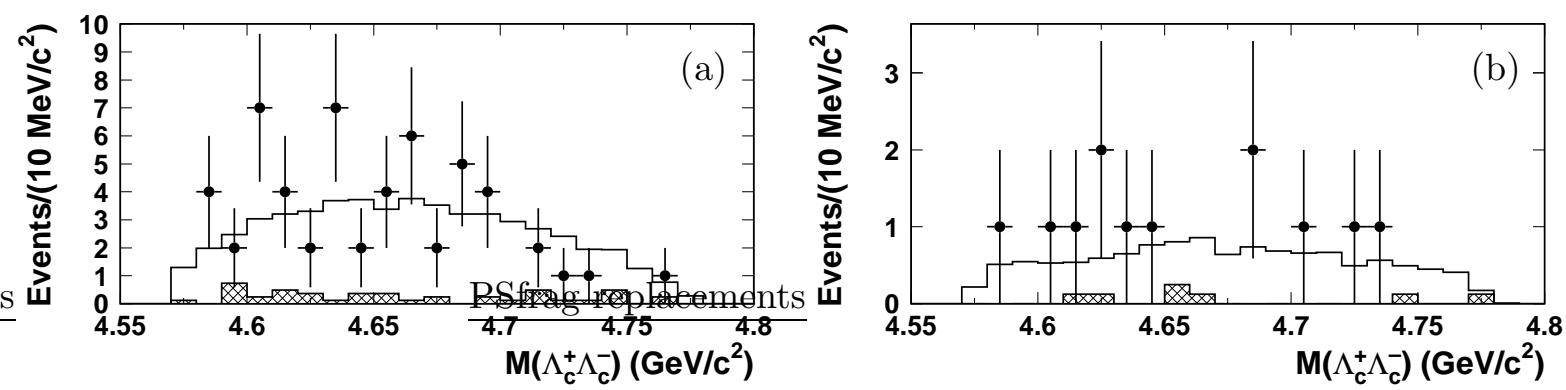

FIG. 3: $\quad M\left(\Lambda_{c}^{+} \Lambda_{c}^{-}\right)$mass distributions for (a) $B^{+} \rightarrow \Lambda_{c}^{+} \Lambda_{c}^{-} K^{+}$and (b) $B^{0} \rightarrow \Lambda_{c}^{+} \Lambda_{c}^{-} K^{0}$ decay candidates in the $B$ signal region. Points with error bars are data. Open histograms - MC for a uniform phase space distribution. Hatched histograms - normalized $\Lambda_{c}^{+}$sideband data.

Figure 3 shows the $M\left(\Lambda_{c}^{+} \Lambda_{c}^{-}\right)$mass distributions for (a) $B^{+} \rightarrow \Lambda_{c}^{+} \Lambda_{c}^{-} K^{+}$decay candidates and (b) $B^{0} \rightarrow \Lambda_{c}^{+} \Lambda_{c}^{-} K^{0}$ decay candidates in the $B$ signal region $\left|\Delta M_{B}\right|<0.015 \mathrm{GeV} / c^{2}$ and $M_{\mathrm{bc}}>5.27 \mathrm{GeV} / c^{2}$. No deviations from phase space distributions are evident.

In summary, we have reported the first measurement of the doubly charmed baryonic $B$ decay $B^{+} \rightarrow \Lambda_{c}^{+} \Lambda_{c}^{-} K^{+}$with a branching fraction of $\left(6.5_{-0.9}^{+1.0} \pm 1.1 \pm 3.4\right) \times 10^{-4}$ and a statistical significance of $15.4 \sigma$, and the $B^{0} \rightarrow \Lambda_{c}^{+} \Lambda_{c}^{-} K^{0}$ decay with a branching fraction of 
$\left(7.9_{-2.3}^{+2.9} \pm 1.2 \pm 4.1\right) \times 10^{-4}$ and a statistical significance of $6.6 \sigma$. These three-body doubly charmed $B$ decay branching fractions are about the same order of magnitude (or slightly

smaller) than the branching fraction of the two-body doubly charmed decay $B^{+} \rightarrow \bar{\Xi}_{c}^{0} \Lambda_{c}^{+}$, which is due to the same $b \rightarrow c \bar{c} s$ quark transition, also observed by Belle [9]. The behavior of these $b \rightarrow c \bar{c} s$ decays is qualitatively different from single charmed baryon decays, where threebody decays have bigger branching fractions than two-body decays. The obtained branching fraction is by five to six orders of magnitude higher than expected from naive estimation for the $B \rightarrow \Lambda_{c}^{+} \Lambda_{c}^{-} K$ decay with color suppression, which is also highly suppressed by phase space [17]. All this needs further experimental and theoretical study.

We thank the KEKB group for excellent operation of the accelerator, the KEK cryogenics group for efficient solenoid operations, and the KEK computer group and the NII for valuable computing and Super-SINET network support. We acknowledge support from MEXT and JSPS (Japan); ARC and DEST (Australia); NSFC and KIP of CAS (contract No. 10575109 and IHEP-U-503, China); DST (India); the BK21 program of MOEHRD, and the CHEP SRC and BR (grant No. R01-2005-000-10089-0) programs of KOSEF (Korea); KBN (contract No. 2P03B 01324, Poland); MIST (Russia); MHEST (Slovenia); SNSF (Switzerland); NSC and MOE (Taiwan); and DOE (USA).

[1] S.A. Dytman et al., (CLEO Collaboration), Phys. Rev. D 66, 091101(R) (2002).

[2] N. Gabyshev et al., (Belle Collaboration), Phys. Rev. D 66, 091102(R) (2002).

[3] N. Gabyshev et al., (Belle Collaboration), Phys. Rev. Lett. 90, 121802 (2003).

[4] N. Gabyshev et al., (Belle Collaboration), arXiv:hep-ex/0409005, submitted to Phys. Rev. Lett.

[5] V. Chernyak and I. Zhitnitsky, Nucl. Phys. B 345, 137 (1990).

[6] P. Ball and H.G. Dosch, Z. Phys. C 51, 445 (1991).

[7] M. Jarfi et al., Phys. Lett. B 237, 513 (1990); M. Jarfi et al., Phys. Rev. D 43, 1599 (1991); N. Deshpande, J. Trampetic and A. Soni, Mod. Phys. Lett. A 3, 749 (1988).

[8] H.Y. Cheng and K.C. Yang, Phys. Rev. D 65, 054028 (2002); ibid D 65, 099901(E) (2002).

[9] R. Chistov et al., (Belle Collaboration), arXiv:hep-ex/0510074, submitted to Phys. Rev. D $(\mathrm{RC})$. 
[10] A. Abashian et al., (Belle Collaboration), Nucl. Instr. and Meth. A 479, 117 (2002).

[11] Z. Natkaniec et al. (Belle SVD2 Group), Nucl. Instr. and Meth. A 560, 1(2006).

[12] Events are generated with the CLEO group's QQ program (http://www.lns.cornell.edu/public/CLEO/soft/QQ). The detector response is simulated using GEANT: R. Brun et al., GEANT 3.21, CERN Report DD/EE/84-1, 1984.

[13] S.L. Zang et al., (Belle Collaboration), Phys. Rev. D 69, 017101 (2004).

[14] B. Aubert et al., (BaBar Collaboration), Phys. Rev. D 72, 052006 (2005).

[15] H. Albrecht et al., (ARGUS Collaboration), Phys. Lett. B 229, 304 (1989); ibid B 241, 278 (1990).

[16] W.-M. Yao et al., (Particle Data Group), J. Phys. G 33, 1 (2006).

[17] H.Y. Cheng, C.K. Chua and S.Y. Tsai, Phys. Rev. D 73, 074015 (2006). 